\title{
Comparative imaging of ectopic mediastinal parathyroid adenoma with magnetic resonance imaging and single photon emission computed tomography/computed tomography: advantages of multimodality imaging
}

Xiangxiang Liu, Zhaowei Meng, Peng Wang, and Qiang Jia

Department of Nuclear Medicine, Tianjin Medical University General Hospital, Tianjin, China

\author{
Received: October 29, 2018 \\ Revised : November 15, 2018 \\ Accepted: January 20, 2019 \\ Correspondence to \\ Zhaowei Meng, M.D. \\ Tel: +86-13132038325 \\ Fax: +86-22-27813550 \\ E-mail:jamesmencius@163.com
}

Figure 1. Scintigraphy and single photon emission computed tomography/computed tomography (SPECT/CT). Parathyroid dual-phase/dual-isotope scintigraphy was performed immediately after the injection of $8 \mathrm{mCi}^{99 \mathrm{~m}} \mathrm{TcO}_{4}$ (A) and performed immediately (B) and 40 minutes postinjection (C) after the injection of ${ }^{99 \mathrm{~m}}$ Tc-sestamibi (methoxyisobutylisonitrile [MIBI]). ${ }^{99 \mathrm{~m}}$ Tc-MIBI scintigraphy showed high tracer uptake in the lesion below the right thyroid lobe, and the lesion became clearer with delayed imaging. The 99mTc-MIBI parathyroid SPECT/CT (DF) showed a solid mass with high tracer uptake in the anterosuperior mediastinum.
A 62-year-old Chinese man was found to have abnormalities during his laboratory examinations with elevated serum calcium (Ca) $(4.08 \mathrm{mmol} / \mathrm{L})$ and alkaline phosphatase (236 U/L) levels and decreased serum phosphorus ( 0.65 $\mathrm{mmol} / \mathrm{L}$ ) and urine calcium (U-Ca) (15.54 mmol/day) levels. The parathyroid hormone (PTH) level (172.00 pmol/L) was increased significantly. The thoracic magnetic resonance imaging (MRI) results suggested that there was a neoplastic lesion in the anterosuperior mediastinum. A whole-body bone scan indicated active bone metabolism. The patient's bone density test results showed that the $\mathrm{T}$ scores of the lumbar vertebrae, the femoral neck, and the total hip were $-2.0,-2.4$, and -2.5 , respectively. The patient had mild osteoporosis. The single photon emission computed tomography/computed tomography (SPECT/CT) results revealed that there was a hyperfunctioning ec-
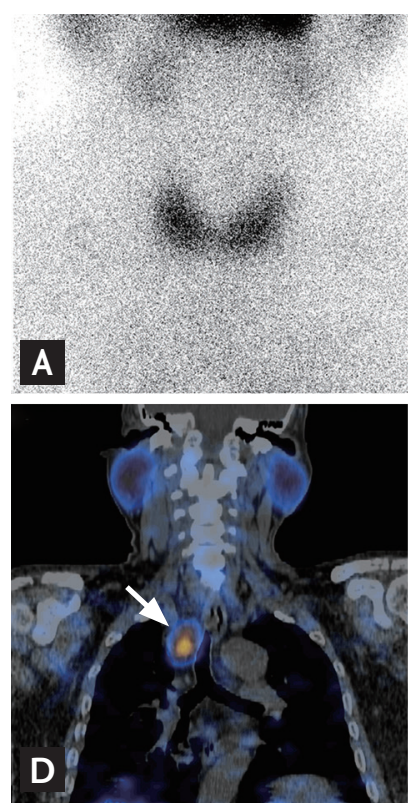

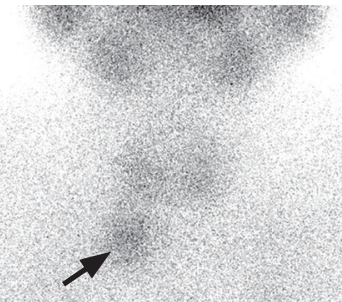

B

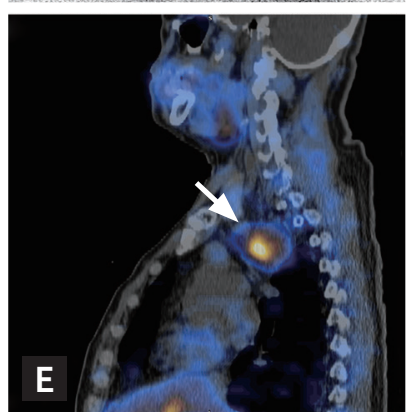

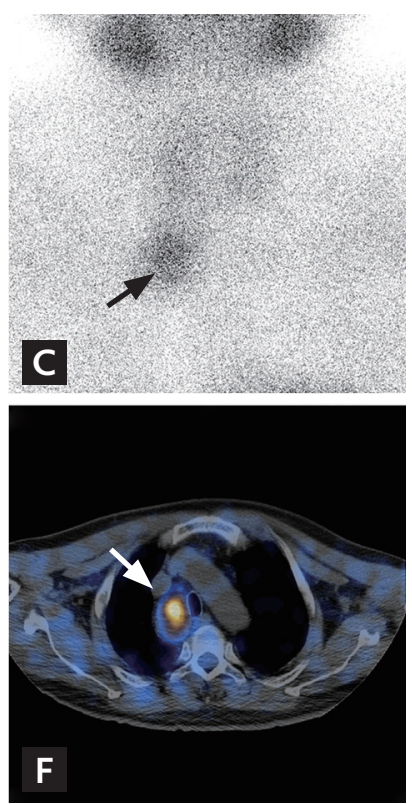



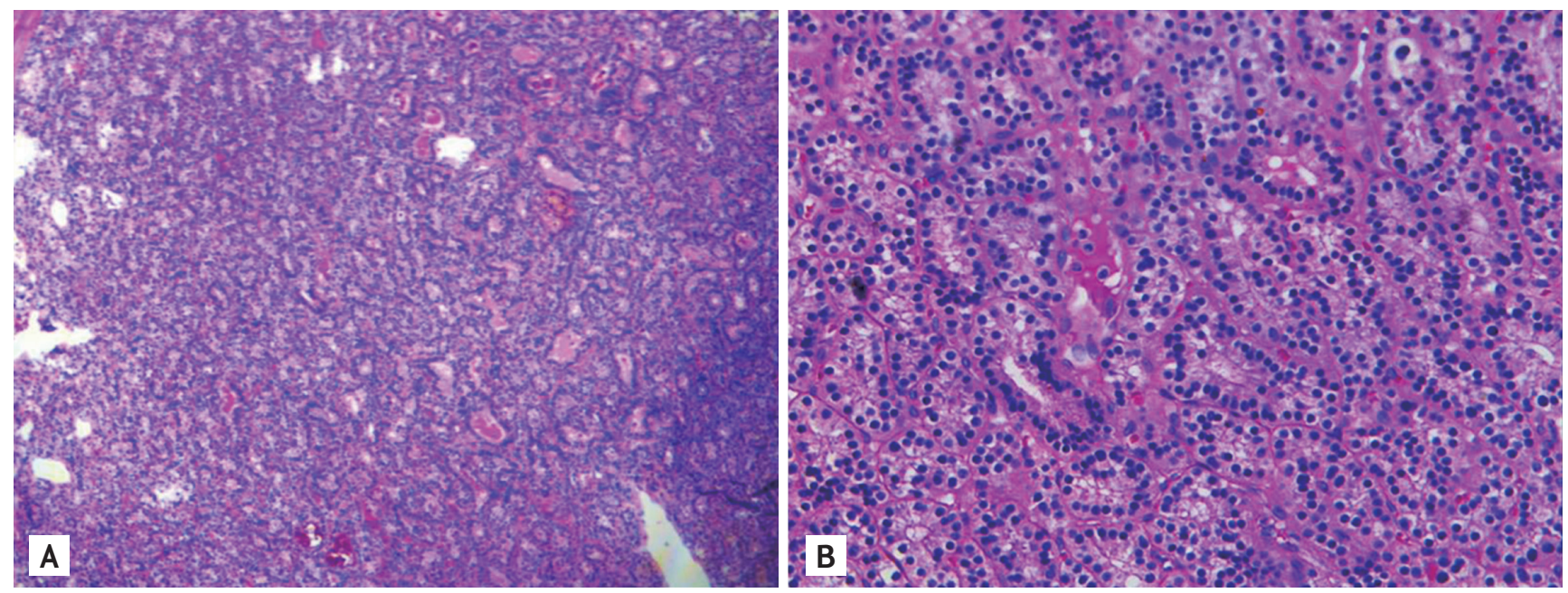

Figure 2. Histopathological findings. (A) The pathological results showed that the tumor cells actively proliferated and had a high growth density $(\mathrm{H} \& \mathrm{E}, \times 4 \mathrm{O})$. (B) The lesion was dominated by bundle-like growth morphology and consisted mainly of small, dark chief cells (H\&E, X100). Immunohistochemical staining showed that the tumor cells were positive for parathyroid hormone and chromogranin A and negative for thyroglobulin, thyroid transcription factor-1, thyrocalcitonin, and synaptophysin, with a Ki-67 index of $20 \%$.

topic parathyroid adenoma (Fig. 1). Afterwards, the patient underwent median sternotomy. The pathological results are presented in Fig. 2. The patient's PTH, Ca, and U-Ca levels all returned to normal postoperatively. All the above findings confirmed a diagnosis of primary hyperparathyroidism due to an ectopic parathyroid adenoma.

Ectopic parathyroid adenoma is an uncommon clinicopathological entity with an incidence rate of $6 \%$ to $20 \%$. Among these cases, approximately $7 \%$ to $45 \%$ of the culprit lesions are located in the anterosuperior mediastinum. Accurate preoperative imaging to locate the ectopic parathyroid adenoma is urgently needed. Different imaging methods have different advantages and deficiencies. For example, ultrasound can hardly detect retrosternal lesions. To assess anterosuperior mediasti- num lesions, MRI has a sensitivity of $82 \%$, while methoxyisobutylisonitrile (MIBI) scintigraphy has a sensitivity of $88 \%$ to $100 \%$. Additionally, CT and MRI can only display anatomical localization. Hybrid imaging, such as the combination of CT/MRI and MIBI planar scintigraphy, can combine both anatomical and functional localizations. This approach increases the sensitivity and provides a more accurate diagnosis. Therefore, multimodality imaging is essential for preoperative guidance, especially in the case of ectopic mediastinal parathyroid adenoma.

The informed consent was waived.

\section{Conflict of interest}

No potential conflict of interest relevant to this article was reported. 